\title{
Glucocorticoid and Mineralocorticoid Insufficiency on Treatment with Tramadol
}

Mansoor Shaikh¹, Catrin Searell² \& Anthony Wilton ${ }^{1}$

${ }^{1}$ Department of Endocrinology, Ysbyty Gwynedd, Bangor, United Kingdom 2Department of Blood Sciences, Ysbyty Gwynedd, Bangor, United Kingdom

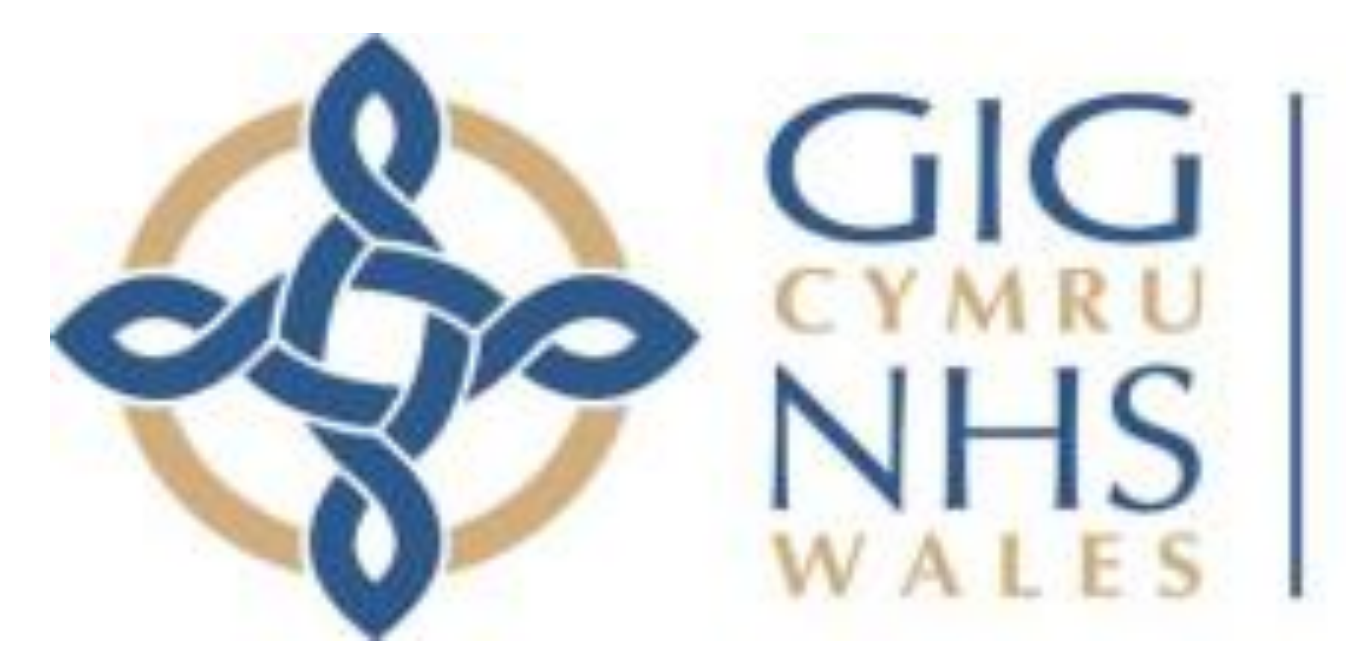

Bwrdd lechyd Prifysgol Betsi Cadwaladr University Health Board

Opioids are known to suppress the hypothalamo-pituitary-adrenal axis. The fourfold increase in the number of opioid prescriptions in the last 20 years has resulted in patients with this side effect and its consequences presenting to specialties other than Endocrinology.

\section{Case History}

A 32 year old female presented to the emergency medicine department with recurrent episodes of collapse with hypotension. She had variously taken tramadol $100 \mathrm{mg}$ qds, morphine sulphate $10-20 \mathrm{mg}$ od and codeine phosphate $60 \mathrm{mg}$ qds for pain due to endometriosis. A random cortisol of $110 \mathrm{nmol} / \mathrm{L}$ led to endocrine referral and opiate-induced hypoadrenalism was considered and investigated.

\section{Investigations}

Results of basal pituitary tests at 09:00h were as follows:

$\begin{array}{lll}\text { Cortisol } & 109 \mathrm{nmol} / \mathrm{L} & (185-624) \\ \text { ACTH } & 3.2 \mathrm{ng} / \mathrm{L} & (7-63) \\ \text { fT4 } & 12.3 \mathrm{pmol} / \mathrm{L} & (7.0-17.0) \\ \text { fT3 } & 3.7 \mathrm{pmol} / \mathrm{L} & (3.5-6.5) \\ \text { TSH } & 1.18 \mathrm{mU} / \mathrm{L} & (0.35-5.50) \\ \text { FSH } & 6.8 \mathrm{IU} / \mathrm{L} & \\ \text { LH } & 12.1 \mathrm{IU} / \mathrm{L} & \\ \text { Prolactin } & 438 \mathrm{mU} / \mathrm{L} & (71-556) \\ \text { IGF-1 } & 14.0 \mathrm{nmol} / \mathrm{L} & (10.0-39.0)\end{array}$

Short synacthen test: (commenced 09:00h, on tramadol 100 mg qds)

\begin{tabular}{|c|c|c|}
\hline Time (min) & Cortisol (nmol/L) & ACTH (ng/L) \\
\hline 0 & 182 & 6.0 \\
\hline 30 & 397 & - \\
\hline
\end{tabular}

Urea \& electrolytes and pituitary MRI were normal.

The following investigations were conducted after withdrawal of opiates:

Cortisol \& ACTH day profile with morphine challenge:

Morphine sulphate $5 \mathrm{mg}$ given 09:00h (morphine half life 1.54.5h)

\begin{tabular}{|c|c|c|}
\hline Time & Cortisol (nmol/L) & ACTH (ng/L) \\
\hline 09:00 & 542 & 43.5 \\
\hline 10:00 & 405 & 10.4 \\
\hline $11: 00$ & 246 & 4.3 \\
\hline $12: 00$ & 154 & 3.0 \\
\hline $13: 00$ & 467 & 13.1 \\
\hline $14: 00$ & 298 & 5.9 \\
\hline 15:00 & 486 & 11.8 \\
\hline
\end{tabular}

Cortisol \& ACTH day profile with tramadol challenge:

Tramadol $100 \mathrm{mg}$ given 09:00h (tramadol half life 6-7h)

\begin{tabular}{|c|c|c|}
\hline Time & Cortisol (nmol/L) & ACTH (ng/L) \\
\hline 09:00 & 175 & 6.1 \\
\hline 10:00 & 140 & 4.7 \\
\hline 11:00 & 91 & 2.4 \\
\hline $12: 00$ & 64 & 2.0 \\
\hline 13:00 & 66 & 3.9 \\
\hline 14:00 & 85 & 6.8 \\
\hline 15:00 & 57 & 3.0 \\
\hline 16:00 & 42 & 2.4 \\
\hline
\end{tabular}

Glucagon test: (commenced at 08:30h) off tramadol for 3 weeks

\begin{tabular}{|c|c|c|c|}
\hline $\begin{array}{c}\text { Time } \\
(\mathrm{min})\end{array}$ & $\begin{array}{c}\text { Gluocse } \\
(\mathrm{mmol} / \mathrm{L})\end{array}$ & $\begin{array}{c}\text { Cortisol } \\
(\mathrm{nmol} / \mathrm{L})\end{array}$ & $\begin{array}{c}\mathrm{GH} \\
(\mu \mathrm{g} / \mathrm{L})\end{array}$ \\
\hline-30 & 4.6 & 425 & 3.13 \\
\hline 0 & 4.4 & 344 & 6.98 \\
\hline 90 & 3.8 & 186 & 0.44 \\
\hline 120 & 6.6 & 140 & 1.63 \\
\hline 150 & 4.3 & 156 & 14.50 \\
\hline 180 & 3.6 & 304 & 25.30 \\
\hline 210 & 3.5 & 279 & 13.30 \\
\hline 240 & 3.9 & 227 & 5.38 \\
\hline
\end{tabular}

Aldosterone $<100 \mathrm{pmol} / \mathrm{L}$ Renin $0.6 \mathrm{nmol} / \mathrm{L} / \mathrm{h}$ (supine) Aldosterone $<100 \mathrm{pmol} / \mathrm{L}$ Renin $1.4 \mathrm{nmol} / \mathrm{L} / \mathrm{h}$ (ambulant)

\section{Discussion}

The results demonstrate hypothalamo-pituitary-adrenal axis suppression with tramadol and morphine, both of which are Mu opiate receptor agonists. The hypo-reninaemic hypoaldosteronism whilst taking tramadol is a novel finding with its relevance to the presentation being unclear (there is evidence that naloxone increases secretion of renin at rest and during exercise). The patient was advised of the need for hydrocortisone if treated with opiates and did so during a recent labour/delivery during which she received morphine analgesia with no untoward effects. 